\title{
The Antidepressant Effect of Chlorella vulgaris on Female Wistar Rats (Rattus norvegicus Berkenhout, 1769) with Chronic Unpredictable Mild Stress Treatment
}

\author{
Gisella Intan Soetantyo ${ }^{*}$, Mulyati Sarto ${ }^{1}$ \\ 1) Faculty of Biology, Universitas Gadjah Mada, Jl. Teknika Selatan, Sekip Utara, Yogyakarta, Indonesia, 55281 \\ *Corresponding author. Tel.: +628179621580. Email address: gisella.intan@yahoo.com
}

\author{
Keywords: \\ Chlorella vulgaris \\ amitriptyline \\ adrenal gland \\ stress \\ depression \\ antioxidant
}

Article history:

Submitted 05/03/2019

Revised 13/06/2019

Accepted 24/07/2019

\begin{abstract}
Depression is a disabling mental disorder, predicted to become the world's number 2 disability by 2020 by the World Health Organization (WHO, 2018). Chronic stress is one of the triggers for depression, causing an imbalance in brain chemicals and antioxidants levels. Although antidepressant is a common treatment, discomforting side effects has compromised its efficacy, prompting the search for alternative medicines. Chlorella vulgaris is a microalgae famous for its excellent protein and antioxidant content. In this study, C. vulgaris $(360 \mathrm{mg} / \mathrm{kg}$ p.o.) potency of antidepressant in chronic unpredictable mild stress (CUMS) model of depression in female rats was evaluated compared to amitriptyline $(2,25 \mathrm{mg} / \mathrm{kg}$ p.o.) for 14 days. Two types of $C$. vulgaris namely cultivation sourced and commercially-sold, were used. Sucrose preference test, forced swim test (FST) and open field test (OFT) were used as depression-like behaviour test to validate $C$. vulgaris effect. Adrenal glands were observed to further understand its effect on the stress organ. The CUMS method produced rats with depressivelike behaviour evidently by reduced body weight, sucrose preference, exploring behaviour in OFT, and increased immobility duration in FST. Furthermore, an increase in adrenal weight, fasciculata zone, and reticularis zone was observed. Both C. vulgaris significantly $(p<0,05)$ reversed depressive-like behaviour in rats subjected to CUMS, but not the size of adrenal glands. This finding indicated both types of $C$. vulgaris has the potential to be an alternative antidepressant but because of the short duration of treatment, it's speculated that $C$. vulgaris may not have exhibited enough difference structurally yet.
\end{abstract}

\section{INTRODUCTION}

In modern times, depression has become a disability that is common in Indonesian society. Research by the Ministry of Health in 2018 states that $6.1 \%$ of people in Indonesia are depressed. Unfortunately, from all recorded patients, only $9 \%$ were treated by professionals (Ministry of Health, 2018). One of the reasons being depression treatment which is still limited to antidepressant drugs that often bring uncomfortable side effects to the body. The common side effects include heart palpitations, insomnia, agitation, headache, and even in extreme cases, stroke (Hedaya, 2011).

One of the alleged cause of depression is chronic stress (Hammen, 2005). Chronic stress can cause a decrease in serotonin and noradrenaline (Natarajan et al., 2015; Goddard et al., 2009). It can also lead to oxidative stress which causes low levels of antioxidants in the body (Srivastava \& Kumar, 2015). In the human body, stress is responded by the adrenal gland. The medulla adrenal will response through the sympatho-adrenomedullary pathway while the adrenal cortex response through cortisol production from the fasciculatory zone via the hypothalamus-pituitary-adrenal axis. Cortisol belongs to the glucocorticoid group which increases blood sugar levels in the body for the provision of instant energy. This energy is used to fight or flee from the stressor (fight or flight response) (Chung et al., 2011). Stress response process generates reactive 
oxygen species (ROS) as a byproduct. A high ROS concentration in the body can result in various poor health conditions, one of them is lipid peroxidation in brain which inhibits the binding of serotonin (Scapagnini, et al., 2012). ROS effects can be reduced by antioxidant.

Serotonin is synthesized from the amino acid tryptophan, while noradrenaline uses the amino acid tyrosine (Hemat, 2004). Both of these amino acids can be obtained from food, one of which is microalgae such as Spirulina sp. and Chlorella sp. (Santhanam, 2015; Gershwin \& Belay, 2008). According to Bewicke \& Potter (2009), Chlorella vulgaris contains tryptophan and tyrosine in high content. In addition, C. vulgaris is also superior in chlorophyll content. Chlorophyll has been studied to have excellent antioxidant activity (Hsu et al., 2013) so that the high chlorophyll in C. vulgaris can give an insight of its potential as a source of antioxidants for humans.

Chlorella vulgaris has long been used as health supplements for humans and are safe for consumption (Klamczynska \& Mooney, 2017). However, it is not yet popular, because of the expensive price due to complicated commercial processing, which is the process of removing cell walls from Chlorella to make it easier to digest (Zheng et al., 2011). Production with traditional cultivation methods omits said process, providing a Chlorella alternative at a more affordable price, but there is no research to determine the difference in performance between the two yet. Seeing the potential for the discovery of alternative medicine to reduce depression from a natural, affordable source, we conducted this study. Two types of Chlorella vulgaris were used (cultivated and commercially sold) to further determine the differences that may exist between the two.

\section{MATERIALS AND METHODS}

\section{Materials}

Twenty-five female Wistar rats (R. norvegicus) aged 12 months with an interval bodyweight of 100-150 grams were used as animal models and were obtained from Laboratory of Advanced Research and Testing Unit IV, University Gadjah Mada, Yogyakarta. C. vulgaris powdered extract was obtained from Blue Green Microalga Technology. Commercially sold $C$. vulgaris tablets were obtained from CNI Sun Chlorella. The antidepressant drug used as a control was amitriptyline (Indofarma). Supporting materials include sucrose powder, ice cubes, distilled water (CV Progo Mulyo), and wood shavings bedding. Animal feed was given in the form of $\mathrm{AD}$ II pellets and reserve osmosis (RO) water was given as drinking water, both ad libitum, meaning the animals can access these freely. Animal models were kept in groups in a cage with each cage containing 5 rats. Rice husk as standard bedding was changed every 2-3 days. Ketamine (Ketalar): xylazine (Interchemie) cocktail with ratio 1: 1 was used as an anaesthetic and euthanasian agent. Materials needed for making histological slides include neutral buffered formalin (NBF) 10\% (CV Progo Mulyo), graded ethanol (CV Progo Mulyo), physiological salt solution $(0.9 \% \mathrm{NaCl})$, Ehrlich Hematoxilin dye, Eosin Y 1\%, distilled water, xylol, toluol, paraffin temperature $57-60^{\circ} \mathrm{C}$, Meyer's albumin, object-glass, and cover glass.

\section{Animal models}

Animal models were adapted in cages and research environment. Acclimation was carried out for 3 days to minimize stress in animals before the research was conducted so the data has good validity. This stage was carried out in the research room II Laboratory of Advanced Research and Testing Unit IV, University Gadjah Mada. Animal models were weighed once a week individually using semi-analytic scales. The weighing was also done before blood sampling for the determination of the anaesthetic dose and before euthanizing for the calculation of the adrenosomatic index.

Blood sampling was carried out for blood glucose level measurement at the beginning of the study after the acclimation process, after stress induction and after the treatment period. Before sampling, animals were anaesthetized first with ketamine (50 mg/kg BW). Blood sampling was done by inserting one end of the micro hematocrit inward medial canthus of the animal's eye, (forming a 30-degree angle to the nose) and was then stored in $1 \mathrm{~mL}$ microtube. Blood glucose measurements were made with a glucose meter (EasyTouch) with corresponding singe use test strips.

\section{Stress Induction by Chronic Unpredictable Mild} Stress (CUMS) Method

Animal models stress was induced by the method CUMS (Hu et al., 2017; Zhang et al., 2014). The stressors given were described as follows:

a. Treatment of cold water, animal models were put in a water tank with a water depth of $25 \mathrm{~cm}$ and a water temperature of $5^{\circ} \mathrm{C}$ for 3 minutes. After treatment, animals were allowed to dry themselves inside a dry cage with fresh woodshavings bedding while illuminated with a lamp to keep the animals warm.

b. Treatment of warm water, animals were put in a water tank with a water depth of $25 \mathrm{~cm}$ and a water temperature of $45^{\circ} \mathrm{C}$ for 3 minutes. After 
treatment for this stressors is the same as cold water treatment.

c. Treatment of wet cages, animals were placed in cages with wet wood shavings bedding as high as $5 \mathrm{~cm}$. This treatment was given for 24 hours. After treatment, the wet bedding was removed and replaced with dry, standard bedding.

d. Dark-light cycle reversal, animals were exposed to light at night (18.00-06.00) and in a dark place during the daytime (06.00-18.00).

e. Sound wave exposure, animals were exposed to the ultrasonic sound for 12 hours.

f. Treatment of tilted cage, animal's cages were tilted $45^{\circ}$ for 24 hours.

g. Food and water deprivation, for 24 hours animals were not fed and the water bottles were removed from the cage. The bedding was replaced beforehand to remove food burrowed in it by the animals. After treatment, feeding was continued normally and water bottles were given back.

These stressors were carried out to all groups except control for 42 days, by giving 1 type of stressor per day. The order of stressors was maintained so that the same stressors did not occur for 2 consecutive days. On day 42, animals underwent behavioural tests to determine their stress state.

\section{Sucrose Preference Test (SPT)}

The test was carried out at the beginning of the study, after stress induction and after the treatment period. The test was started by habituation of animals to two drinking bottles for 3 days. After 3 days, one of the bottles was filled with $2 \%$ sucrose solution and the other was filled with RO water. Animal cages were then not equipped with drinking bottles for 12 hours, after which both bottles were provided together. The position of the bottle was exchanged every 12 hours and the consumption of sucrose solution and $\mathrm{RO}$ water was measured after 24 hours. Sucrose preference was then calculated based on the following formula: Sucrose preference $(\%)=$ (Sucrose solution consumption / Total water and solution consumption) $\mathrm{mL} \times 100 \%$ (Shukkoor et al., 2016)

\section{Open Field Test (OFT)}

The test was done after stress induction and after the treatment period and refers to the method used by Shukkoor et al. (2016) with changes. Animals were placed in the arena of the lidless opaque plastic box $(30 \mathrm{~cm} \times 60 \mathrm{~cm}$ with height $40 \mathrm{~cm}$ ) without bedding individually for 10 minutes. The movements and behaviours of the animals were recorded using a camera during this period. After 10 minutes, the animal was removed from the arena.

\section{Forced Swim Test}

This test was done immediately after OFT was done. This protocol follows the literature from Slattery \& Cryan (2012). This test consists of two sessions separated by a 24 hour period. At each session, animals were placed in a water tank made of clear acrylic (diameter $20 \mathrm{~cm}$ and height $50 \mathrm{~cm}$ ), which was then filled with water at $26^{\circ} \mathrm{C}$. Water filled the cylinder to a depth of $25 \mathrm{~cm}$ so that the entire body of the animal can be submerged and to prevent the animal's front limbs from reaching the edge of the container and running away.

The day before the test, 15 minutes of the pretest was carried out to habituate animals to the test environment. The next day, the animal was individually put on a water tank to swim for 5 minutes and immobility time was observed. The animal was then transferred to a dry cage containing fresh bedding and illuminated by the lamp as warmer. After the animal's body was relatively dry, it was returned to their respective cages. Faecal boli that may be present in the water tank were removed, and water was replaced every 3 test or if necessary.

\section{Per oral Treatment}

This study was designed randomly with 5 treatment groups. The treatment group consisted of 1 control group, 1 stress control group, 1 positive control group treated with $2,25 \mathrm{mg} / \mathrm{kg}$ BW Amitriptyline and 2 groups of variations in treatment, namely treatment of cultivated $C$. vulgaris and treatment of commercial C. vulgaris, both given at $360 \mathrm{mg} / \mathrm{kg} \mathrm{BW}$ dosage. Each group consists of 5 replications. The duration of treatment is 14 days after stress induction. Treatment was given every morning between 9:00 and 11:00.

\section{Euthanasia and surgery}

On day 56 (or after the treatment period), the animals were terminated by the exsanguination method. Adrenal glands were cleaned and used to find out the adrenosomatic index according to the formula: adrenosomatic index $=$ (total organ weight / total body weight) gram x 100\% (Avinashe, 2013). Adrenal glands were then moved to neutral buffer phosphate (NBF) 10\% solution to fixate it. Then the histological preparations were made with Hematoxylin-Eosin staining.

\section{Data Analysis}

Obtained data were analysed using one-way ANOVA with a confidence level of $95 \%(\alpha<0,05)$ and Duncan Multiple Range Test as post-hoc test. Statistical analysis was done with SPSS 23 program and graphs needed were made using Microsoft 


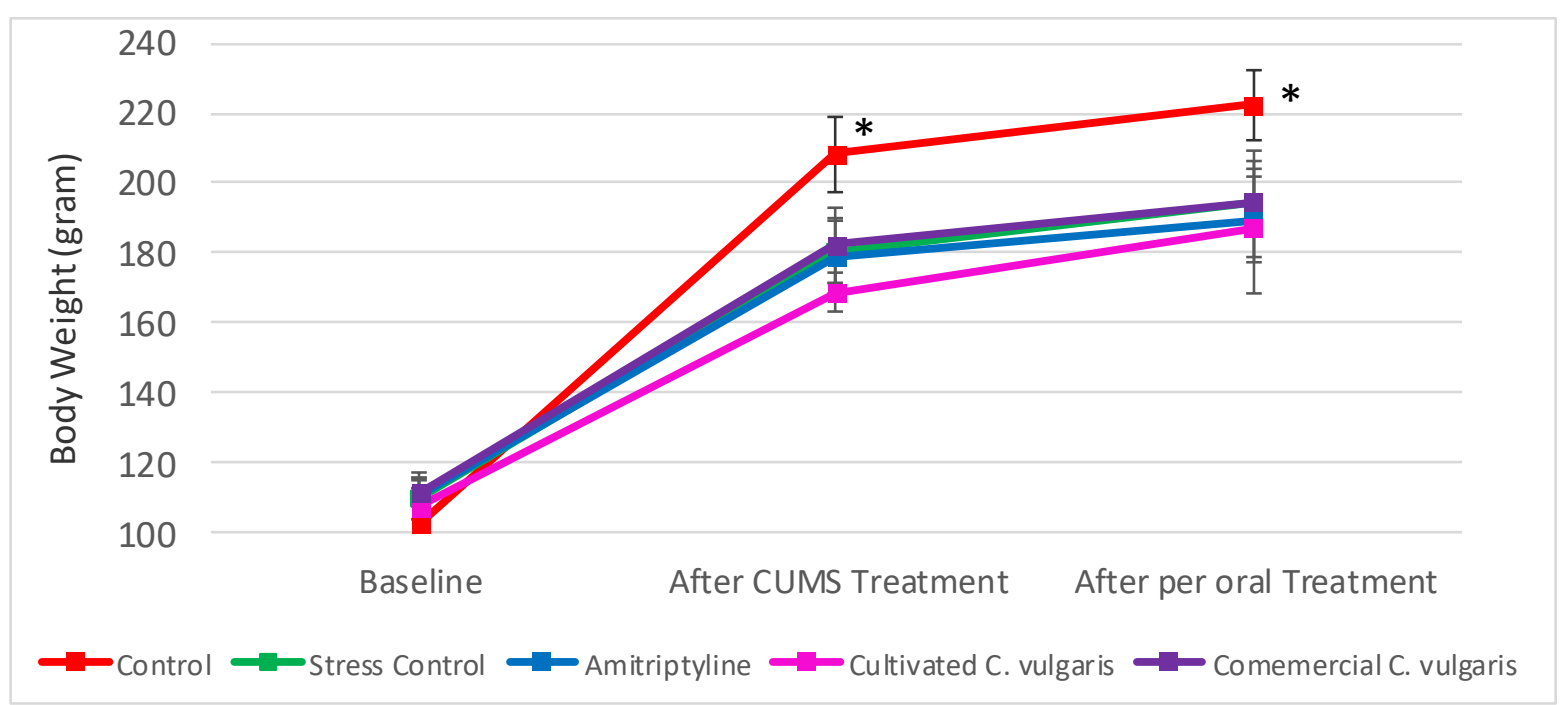

Figure 1. Weight gain in female Wistar rats during the study. ${ }^{*} p<0.01$, when compared with CUMS, treated groups; one-way ANOVA followed by Duncan post-hoc test.

Excel. Qualitative data in the form of roaming videos were analysed using idTracker software (created by Perez-Escudero et al. (2014) and can be downloaded free via the web http:// www.idtracker.es /) to see trajectory patterns made by the animal during the test period.

\section{RESULTS AND DISCUSSION}

This study has passed the required ethical feasibility test by the Animal Ethics Committee of Universitas Gadjah Mada before the study was conducted with a certificate number: 00042/04/ LPPT/VI/2018

\section{Body Weight}

Bodyweight is data that can be linked to animal physiological conditions. In this study, animal body weight was weighed at the beginning of the study, after CUMS treatment and after per oral treatment. Changes that might occur were observed.

Figure 1 showed that all group experienced weight gain from the beginning of the study until the end of CUMS treatment, with control group having a significant difference $(\mathrm{F}=8.504 ; \mathrm{df}=4.18 ; p<0.01)$ compared to all other groups. This can be caused by stress in CUMS treated groups which caused the tendency to consume fewer amounts of food (hypophagia) (Rabasa \& Dickson, 2016). Stress will cause the body to release various stress hormones like $\mathrm{CRH}, \mathrm{ACTH}$, and cortisol which in turn will prepare the body for the "flight or fight" condition. This condition includes increased blood pressure, gluconeogenesis rate, and blood supply to the muscles, heart, and brain. Therefore, organ activities that require energy but not related to this response such as digestive and reproductive organs will be temporarily inhibited (Yau \& Potenza, 2014). Per oral treatment did not cause a difference afterwards compared to the stress control group (Figure 1). This could be due to the short period of treatment which did not provide enough time for structural change to develop. Furthermore, the adaptation to stressors in the form of increased calorie use efficiency in the stress control group (Rabasa \& Dickson, 2016) and late age factor could also play a role in means of a slower metabolism, which could explain the minimum weight gain observed (Barnett, 2017).

\section{Blood Glucose Levels}

Blood glucose level can describe stress status in rat. Figure 2 showed that the stress treatment given did not have a significant effect on blood glucose.

During the initial period of the study until stress conditions were reached, all groups experienced elevated blood glucose levels with insignificant differences. After going through per oral treatment according to the research design, the control and stress control group had a decreased blood glucose value whereas the remaining treatment group experienced an increase.

In theory, stress can increase glucose levels acutely through the work of the epinephrine and cortisol hormones which stimulate liver glycogenolysis and gluconeogenesis. These processes will return to normal conditions (homoeostasis) after the stressor ends (Preiser, 2016) so that an increase in blood glucose is also possible but when the measurement was done, the animal has been able to restore its blood glucose level to its initial state.

Besides that, it was found that microalgae treatments can increase blood glucose levels, seen from the elevated value that happened after per oral 


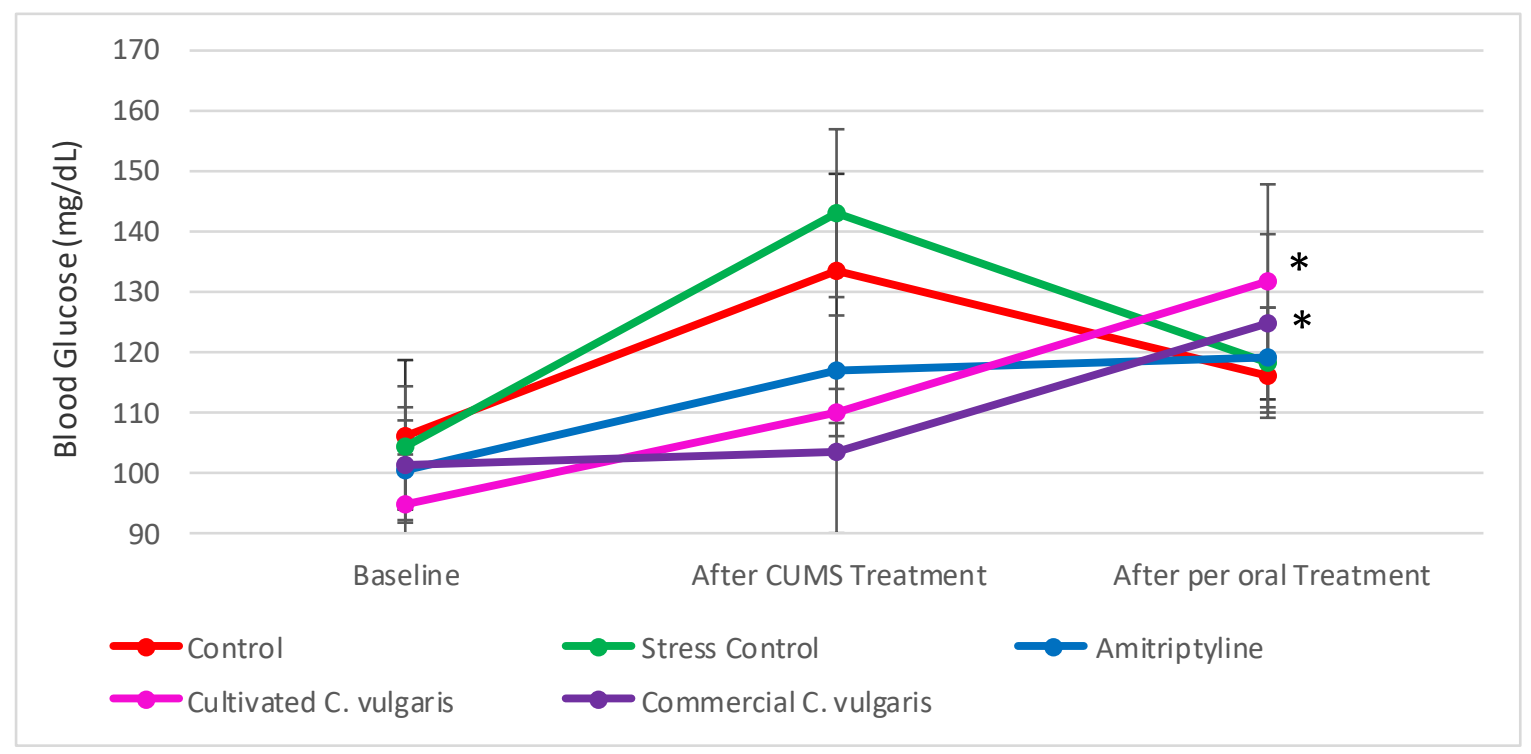

Figure 2. Blood glucose level of female Wistar rats at the beginning of the study, after CUMS treatment and after per oral treatment. ${ }^{*} p<0.05$ when compared to its respective group on its previous period (Baseline and after CUMS treatment).

period $(\mathrm{F}=10.829 ; \mathrm{df}=2.12 ; p<0.05)$. According to Safi et al. (2014), the carbohydrate content of $C$. vulgaris is quite high, at around $55 \%$ of its dry weight, with the predominance of carbohydrates in the form of sugar as a constituent of its cell walls like galactose, glucose, xylose, arabinose, and mannose. Cultivated C. vulgaris provide a higher increase than commercial $C$. vulgaris because cultivated $C$. vulgaris are still intact with the cell wall, whereas in the commercial one which has been factory processed, this cell wall has been removed.

\section{Sucrose Preference}

The preference of animals against sucrose was measured using the sucrose preference test (SPT). Low preference reflects anhedonia behaviour, while high preference is generated by model animals under normal conditions.

On Figure 3, it can be seen that the sucrose preference of the control group stayed relatively high, unlike the other four groups which experienced a decline. This shows the anhedonia behaviour on groups with CUMS treatment which is one of the signs of depression.

After going through per oral treatment, the sucrose preference of animal in the treatment group of both amitriptyline and microalgae return to its original state and approached sucrose preferences of the control group. The animal in the stress control group had its sucrose preference decreased and differed significantly from other groups and even that group itself in the previous week $(\mathrm{F}=7.33$; $\mathrm{df}=4.18 ; p<0.01)$, indicating that sucrose preference in this group continues to decline. Rats naturally have a tendency to consume sweet drinks, so that a decrease in sucrose preference can be assumed as anhedonic behaviour (Serchov et al., 2016).

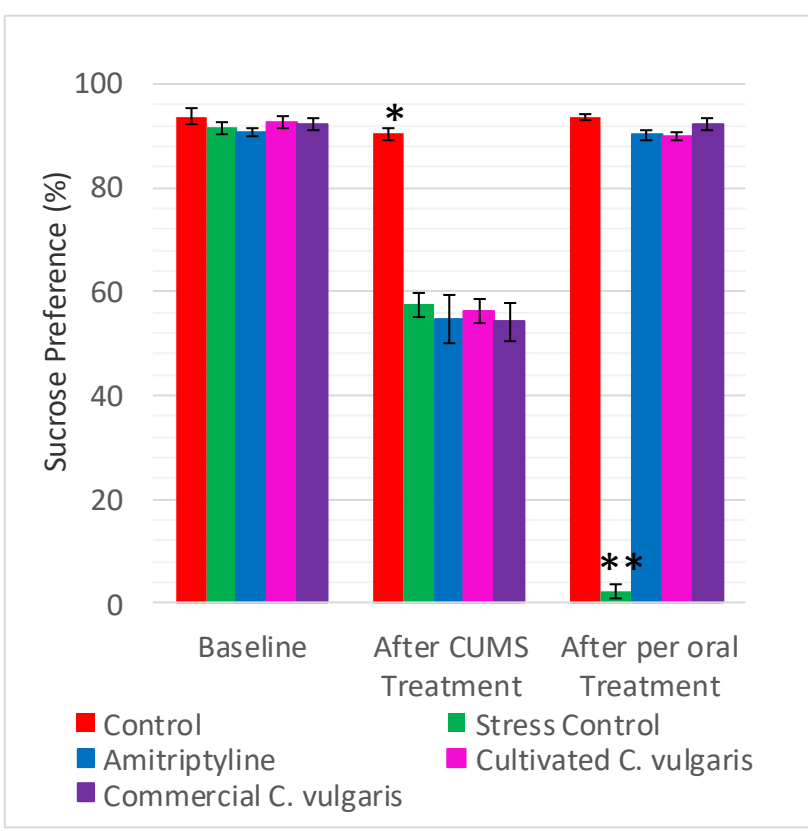

Figure 3. Sucrose preference of female Wistar rats at the beginning of the study, after CUMS treatment and after per oral treatment. * $p<0.01$ compared with CUMS treated groups; ${ }^{* *} p<0.01$ compared with control and per oral groups; one-way ANOVA followed by Duncan posthoc test.

Amitriptyline was chosen because of its efficiency compared to other types of antidepressant drugs in treating depression (Cipriani et al., 2018). Amitriptyline works by increasing the concentration of serotonin and norepinephrine neurotransmitters at synapses (Gupta et al., 2015). When compared with groups treated with cultivated C. vulgaris and commercial C. vulgaris, group treated with cultivated C. vulgaris had a similar preference while the group 


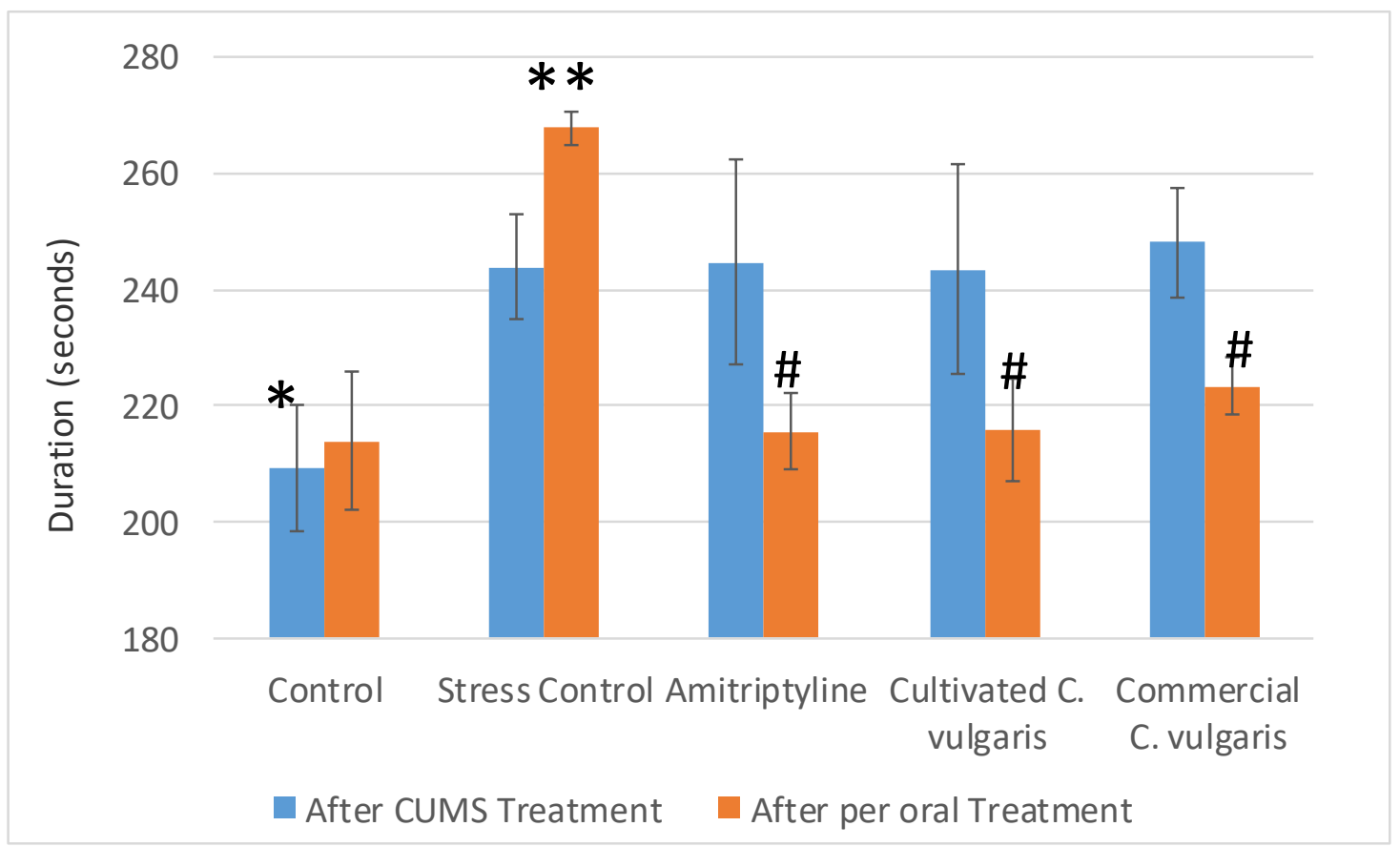

Figure 4. Immobile duration during FST on female Wistar rats after CUMS treatment and per oral treatment. $* p<0.01$ compared with stress control and per oral treated groups; ${ }^{* *} p<0.01$ compared with control and per oral treated groups ${ }^{\#} p$ $<0.01$ compared with the same group respectively after CUMS treatment; one-way ANOVA followed by Duncan posthoc test.

treated with commercial C. vulgaris had a higher preference value. This indicates that both type $C$. vulgaris can reduce anhedonia behaviour in animal models.

\section{Forced Swim Test}

Forced Swim Test (FST) is a common behavioural test on rodents, conducted with the aim of knowing whether a substance or a treatment has the antidepressant effect.

Based on the results on Figure 4, it was discovered that the control group had significantly $(\mathrm{F}=5.415 ; \mathrm{df}=4.18 ; \quad p<0.01)$ shorter duration of immobility compared with other groups. This duration has not differed much on either stress period or treatment period, which showed the consistency of animal conditions in this group throughout the study.

The negative control group had an increased immobile duration from the stress period to the treatment period. This was different from the group with per oral treatment, all of which showed a decline $(\mathrm{F}=28.576 ; \mathrm{df}=4.18 ; p<0.01)$. Immobile behaviour on FST indicates despair in rodents when it realized its effort to escape from the tank is in vain, and this behaviour constitutes synonymous behaviour observed in depressed humans (Belovicova et al., 2017). Amitriptyline treatment as an antidepressant caused a decrease in the duration of immobility. The same decline was observed in the group with microalgae treatment. These results indicate both type C. vulgaris has the potential to reduce the duration of immobility behaviour on FST.

\section{Open Field Test}

Open Field Test (OFT) has been used extensively to measure anxiety behaviour in rodents. The principle of this test involves experimental animals in an unknown environment and observing the response of animals to this condition. Rodents generally choose to be on the edge and sides of the arena, avoiding the centre, unless given a substance with an anxiolytic or similar effect. This test was carried out in this research, in addition to give a more complete picture of the psychological condition of experimental animals, also because the symptoms of anxiety and depression are often times similar to each other (Tiller, 2013). The roaming of the animals was projected into a pattern and each pattern was quantified following the scale provided in Table 1.

From Figure 5 it was stated that control group has a lower roaming value than other groups, significantly in stress conditions $(\mathrm{F}=24.657$; $\mathrm{df}=4.18$; $p<0.01)$ but not significant for animals with amitriptyline treatment at the end of study $(\mathrm{F}=15.933 ; \mathrm{df}=4.18 ; p<0.01)$.

Chronic stress generally decreases the level of animal activity in the new environment, and this symptom can be eliminated by using antidepressants (Wang et al., 2014). Group treated with microalgae produce more complex roaming patterns compared to the stress control group, but not significant 
Table 1. Quantification of the trajectory pattern of rats in the OFT

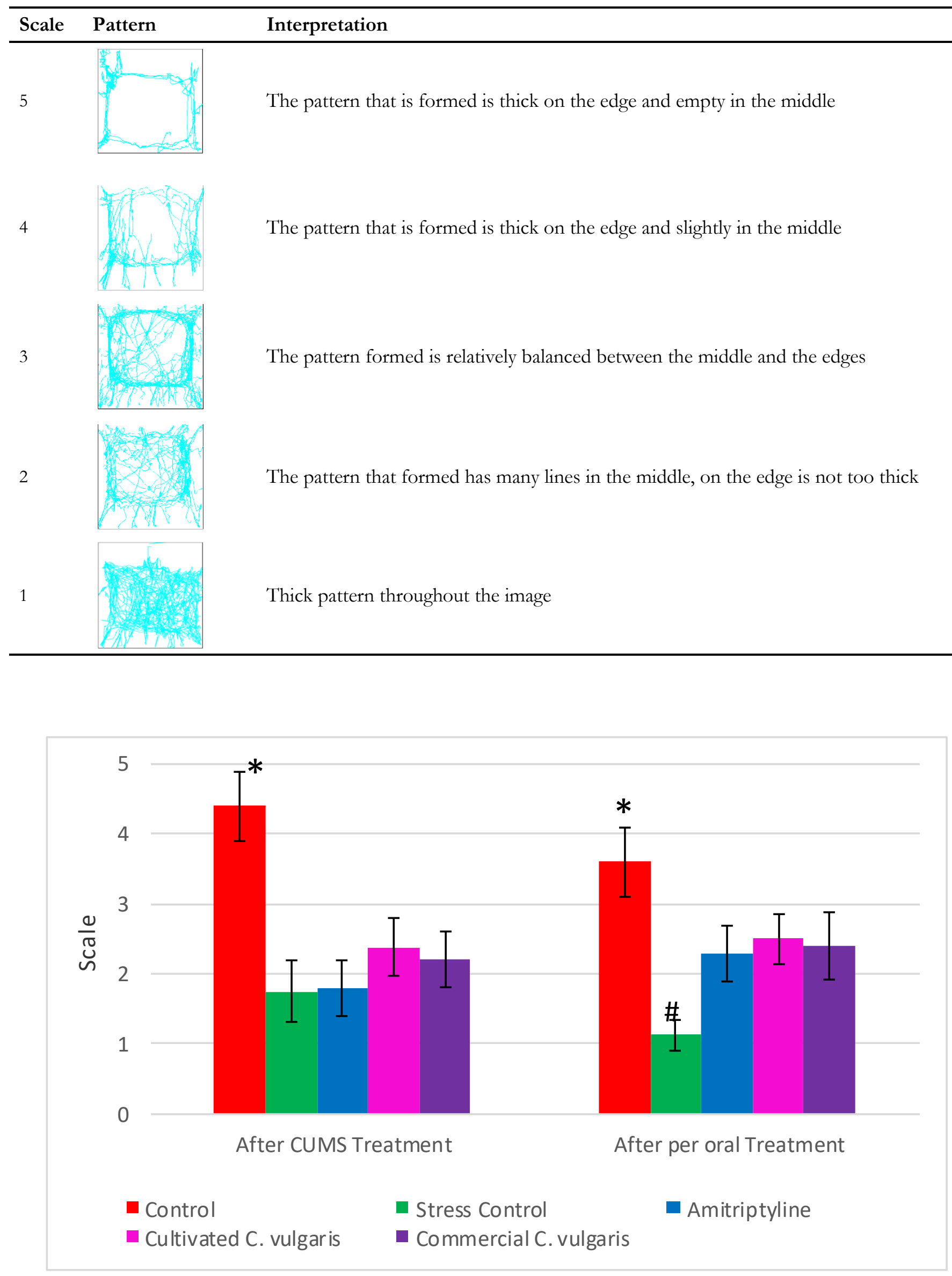

Figure 5. Comparison of the roaming behaviour of female Wistar rats in OFT after CUMS treatment and per oral treatment. ${ }^{*} p<0.01$ compared with stress control and per oral treated groups; ${ }^{*} p<0.01$ compared with control and per oral treated groups. 
Table 2. Adrenal gland of female Wistar rats after 56 days of study.

\begin{tabular}{|c|c|c|c|c|c|}
\hline Parameters & Control & $\begin{array}{l}\text { Stress } \\
\text { Control }\end{array}$ & Amitriptyline & $\begin{array}{l}\text { Cultivated } \\
\text { C. vulgaris }\end{array}$ & $\begin{array}{l}\text { Commercial } \\
\text { C. vulgaris }\end{array}$ \\
\hline Adrenal Weight (grams) & $0.045 \pm 0.012^{\mathrm{x}}$ & $0.067 \pm 0.005^{z}$ & $0.052 \pm 0.004 \mathrm{xy}$ & $0.055 \pm 0.01 \mathrm{xyz}$ & $0.063 \pm 0.008 \mathrm{yz}$ \\
\hline Adrenosomatic Index (\%) & $0.020 \pm 0.006^{x}$ & $0.035 \pm 0.004 y$ & $0.028 \pm 0.003 y$ & $0.029 \pm 0.002 y$ & $0.032 \pm 0.004 y$ \\
\hline Zona Glomerulosa & $11.76 \pm 0.91 \mathrm{yz}$ & $12.40 \pm 1.71^{z}$ & $10.53 \pm 1.33^{\mathrm{xy}}$ & $10.34 \pm 0.55^{\mathrm{xy}}$ & $9.66 \pm 0.75^{x}$ \\
\hline Zona Fasciculata & $70.66 \pm 2.3^{x}$ & $75.80 \pm 4.18 \mathrm{y}$ & $77.27 \pm 2.59 \mathrm{y}$ & $78.49 \pm 2.25 y$ & $76.87 \pm 2.37 \mathrm{y}$ \\
\hline Zona Reticularis & $17.64 \pm 2.02 y$ & $11,66 \pm 1,07 x$ & $11.30 \pm 1.01^{\mathrm{x}}$ & $10.45 \pm 1.09^{\mathrm{x}}$ & $12.57 \pm 1.18^{x}$ \\
\hline Cortex Medulla Ratio & $48.23 \pm 5.28^{x}$ & $51.73 \pm 7.18^{\mathrm{x}}$ & $47.45 \pm 6.1^{x}$ & $51.73 \pm 4.15^{\mathrm{x}}$ & $52.76 \pm 4.83^{x}$ \\
\hline
\end{tabular}

$\mathrm{x}, \mathrm{y}, \mathrm{z}$ : different notation indicates significant difference at $p<0.05$

compared to the amitriptyline treatment group. This could mean that microalgae treatment both cultivated and commercial has the potential to increase motor activity in the OFT.
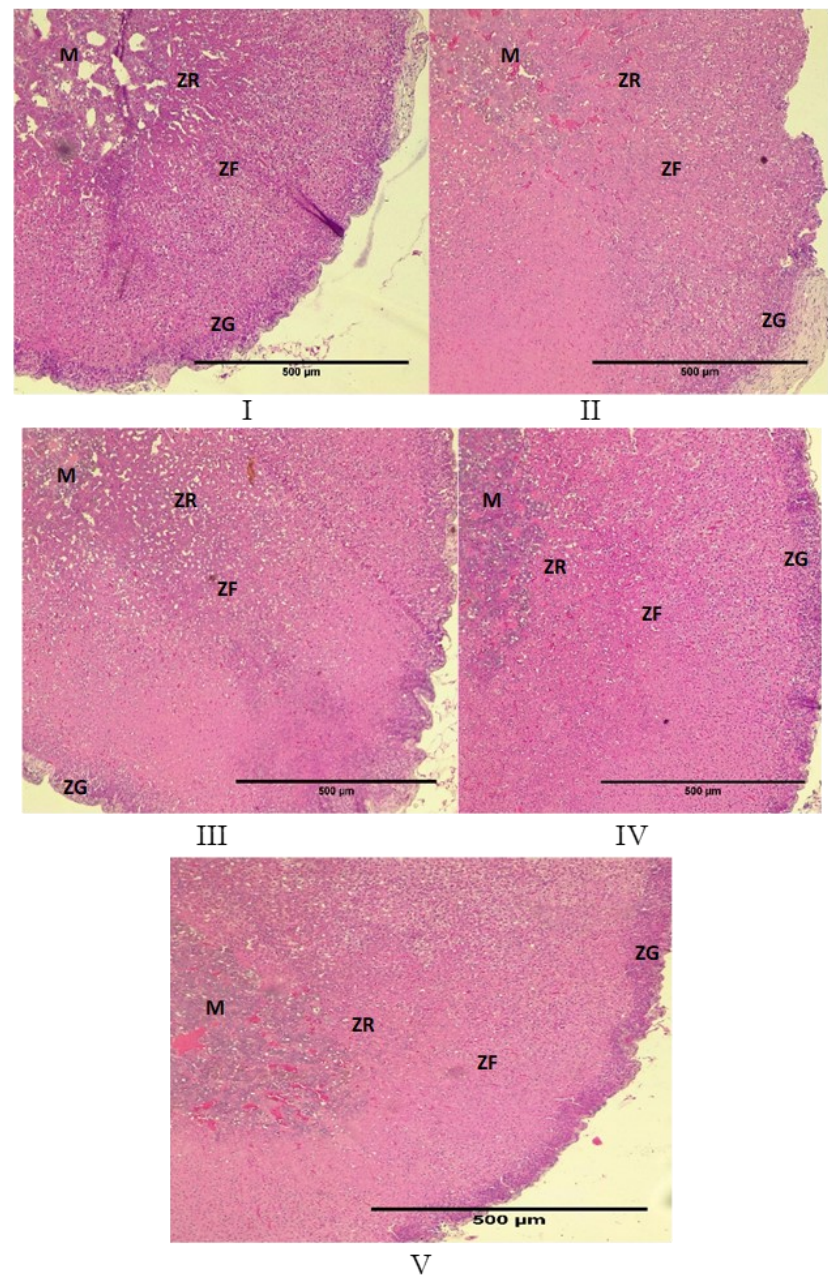

Figure 6. Histological observation of adrenal gland (100 magnification, HE stain). (I: control; II: stress control; III: amitriptyline; IV: cultivated C. vulgaris; V: commercial C. vulgaris. M: medulla; ZR: zona reticularis; ZF: zona fasciculata; ZG: zona glomerulosa).

\section{Adrenal Glands}

Stress experienced by animals can increase its adrenal gland weight due to the role of this gland in producing the hormone ACTH. This increase in weight is caused by hyperplasia and adrenal hypertrophy, reflecting the overwork of this gland (Ulrich-Lai et al., 2006).

Table 2 stated that the adrenosomatic index of animals in the control group was significantly lower than the other groups $(\mathrm{F}=3.987 ; \quad \mathrm{df}=4.18$; $p<0.05)$. The negative control group has the largest index, however, not significant with per oral treated group. No significant results between stress control groups and the amitriptyline treatment group were possible because the drug had not taken effect structurally. Zoladz et al. (2013) found a decreased adrenal weight compared to the stressed rat after 1 month period of amitriptyline administration. The same fact could possibly be the reason why both type C. vulgaris failed to give a significant effect.

Based on histological observations, it was found that the percentage of zona fasciculate in the group with CUMS treatment was significantly greater $(\mathrm{F}=4.473 ; \mathrm{df}=4.18 ; p<0.05)$ than the control group, but zona reticularis $(\mathrm{F}=18.05 ; \mathrm{df}=4.18$; $p<0.05$ ) was significantly smaller (Table 2, Figure 6). Zona fasciculata is where the hormone cortisol formed, which is stimulated by the ACTH hormone from the anterior pituitary. Cortisol plays a role in stress responses such as increasing blood pressure and energy metabolism. Chronic stress causes an increase in cortisol production so that the fasciculata zone enlarges (Ulrich-Lai et al., 2006). Zona reticularis plays a role in reproduction system by producing sex hormone. Stressful events may lead to the inhibition of bodily systems which do not directly support survivability, causing energy supply to these systems to reduce until the stress has passed away (Yau \& Potenza, 2014). The ratio between the cortex and medulla between groups is not significantly different. This is presumably due to enlargement of the cortex followed by enlargement of the adrenal medulla. The adrenal medulla is a place for the conversion of tyrosine amino acids into catecholamines such as epinephrine and norepinephrine. These catecholamines function in the fight or flight response and cause reactions in the body such as increased heart rate, blood pressure, narrowing of blood vessels in the skin and the gastrointestinal system and increased metabolism. 
Prolonged stress will cause over-activation of the system and can cause an increase in mass in the adrenals (Ulrich-Lai et al., 2006).

Brain cells are prone to damage from lipid peroxidation because most of the neuronal cell membranes are composed of unsaturated fatty acids which are the main substrates of ROS. Endogenous antioxidant defence can naturally reduce the production of ROS but chronic stress from the CUMS method is able to damage the endogenous antioxidant defences. This caused an increase in lipid peroxidation and has been studied to be one of the contributing factors to depression (Che et al., 2015). C. vulgaris has been studied to have high antioxidant content (Saranya et al., 2014) and is thought to be the cause of the antidepressant effect observed in this study. Furthermore, C. vulgaris is rich in tryptophan and tyrosine (Santhanam, 2015), the precursor to the neurotransmitter serotonin and noradrenaline (Hemat, 2004). These neurotransmitter's low concentration in the brain has been linked to depression as well (Lopez-Munoz \& Alamo, 2011; Moret \& Briley, 2011)

\section{CONCLUSIONS}

The conclusion of this study is the administration of C. vulgaris $360 \mathrm{mg} / \mathrm{kgBW}$ p.o. in female Wistar rats for 14 days after the CUMS period for 42 days was able to reduce depression-like behaviours, but was not able to revert adrenal glands size back to normal. C. vulgaris source did not make a notable difference to the antidepressant effects in this study. The potential of C. vulgaris as an antidepressant was thought to originate from its high antioxidant content which able to reduce oxidative stress in brain cells.

\section{ACKNOWLEDGEMENTS}

This study was made possible by the help of the Laboratory of Advanced Research and Testing Unit IV, University Gadjah Mada, Yogyakarta. The writers also wish to express their sincerest gratitude to those who have helped along in this research.

\section{REFERENCES}

Avinashe, A.M., 2013, Role of adrenal gland in the gonadosomatic index of male Calotesversicolor (Boulenger), Vidyabharati International Interdisciplinary Research Journal 2(1), 71-75.

Barnett, S.A., 2017, The Rat: A Study in Behavior, Routledge, NY, pp. 124
Belovicova, K., Bogi, E., Csatlosova, K., \& Dubovicky, M., 2017, Animal tests for anxietylike and depression-like behavior in rats, Interdisciplinary Toxicology 10(1), 40-43.

Bewicke, D. \& Potter, B.A., 2009, Chlorella: The Emerald Food, Ronin Publishing, California, pp. $1-26$.

Che, Y., Zhou, Z., Shu, Y., Zhai, C., Gong, S., Cui, Y. \& Wang, J.F., 2015, Chronic unpredictable stress impairs endogenous antioxidant defense in rat brain, Neuroscience Letters 584, 208-13.

Chung, S., Hoon Son, G. \& Kim, K., 2011, Circadian rhythm of adrenal glucocorticoid: Its regulation and clinical implications, Biochimica et Biophysica Acta (BBA) - Molecular Basis of Disease 1812 (5), 581-591.

Cipriani, A., Furukawa, T.A., Salanti, G., Chaimani, A., Atkinson, L.Z., Ogawa, Y., Leucht, S., Ruhe, H.G., Turner, E.H., Higgins, J.P., Egger, M., Takeshima, N., Hayasaka, Y., Imai, H., Shinohara, K., Tajika, A., Ioannidis, J.P.A. \& Geddes, J.R., 2018, Comparative efficacy and acceptability of 21 antidepressant drugs for the acute treatment of adults with major depressive disorder: a systematic review and network meta-analysis, The Lancet 17, 32802-7.

Goddard, A.W., Ball, S.G., Martinez, J., Robinson, M.J., Yang, C.R., Russell, J.M. \& Shekhar, A., 2009, Current perspectives of the roles of the central norepinephrine system in anxiety and depression, Depression and Anxiety 27(4), 339350.

Gupta, G., Jia, T.J., Woon, L.Y., Chellappan, D.K., Candasamy, M. \& Dua, K., 2015, Pharmacological Evaluation of Antidepressant -Like Effect of Genistein and Its Combination with Amitriptyline: An Acute and Chronic Study, Advances in Pharmacological Sciences 2015, 1-6.

Hammen, C., 2005, Stress and depression, Annual Review of Clinical Psychology 1, 293-319.

Hedaya, R.J., 2011, The Antidepressant Survival Guide: The Clinically Proven Program to Enhance the Benefits and Beat the Side Effects of Your Medication, Three Rivers Press, N.Y.

Hemat, R.A.S., 2004, Principles of Orthomolecularism, Urotext, London, p 37.

Hsu, C., Chao, P., Hu, S. \& Yang, C., 2013, The Antioxidant and Free Radical Scavenging Activities of Chlorophylls and Pheophytins, Food and Nutritions Sciences 4, 1-8. 
Hu, C., Luo, Y., Wang, H., Kuang, S., Liang, G., Yang, Y., Mai, S. \& Yang, J., 2017, Reevaluation of the interrelationships among the behavioral tests in rats exposed to chronic unpredictable mild stress, Plos One 12(9), e0185129.

Klamczynska, B. \& W. D. Mooney. 2017. Sustainable Protein Sources. Academic Press. California. pp 327-33.

Lopez-Munoz, F. \& Alamo, C., 2011, Neurobiology of Depression, CRC Press, California, pp. 143-159.

Moret, C. \& Briley, M., 2011, The importance of norepinephrine in depression, Neuropsychiatric Disease and Treatment 7(Suppl 1), 9-13.

Natarajan, R., Northrop, N. A. \& Yamamoto, B. K., 2015, Protracted effects of chronic stress on serotonin-dependent thermoregulation, Stress 18(6), 668-676.

Perez-Escudero, A., Vicente-Page, J., Hinz, R.C., Arganda, S. \& de Polavieja, G.G., 2014, idTracker: tracking individuals in a group by automatic identification of unmarked animals, Nature Methods 11(7), 743-8.

Preiser, J, 2016, The Stress Response of Critical Illness: Metabolic and Hormonal Aspects, Springer, California.

Rabasa, C. \& Dickson, S.L., 2016, Impact of stress on metabolism and energy balance, Behavioral Sciences 9, 71-77.

Safi, C., Zebib, B., Merah, O., Pontalier, P. \& VacaGarcia, C., 2014, Morphology, composition, production, processing and applications of Chlorella vulgaris: A review, Renewable and Sustainable Energy Reviews 35, 265-78.

Santhanam, R., 2015, Nutritional Freshwater Life, CRC Press, California, pp.9.

Saranya, C., Hemalatha, A., Parthiban, C., Anantharaman, P., 2014, Evaluation of Antioxidant Properties, Total Phenolic and Carotenoid Content of Chaetoceros calcitrans, Chlorella salina and Isochrysis galbana, International Journal of Current Microbiology and Applied Sciences 3(8), 365-377.

Scapagnini, G., Davinelli, S., Drago, F., De Lorenzo, A. \& Oriani, G., 2012, Antioxidants as Antidepressants: Fact or Fiction?, CNS Drugs 26(6), 477-90.

Serchov, T., van Calker, D., \& Biber, K., 2016, Sucrose Preference Test to Measure Anhedonic Behaviour in Mice, Bio-protocol 6 (19), e1958. DOI: 10.21769/BioProtoc.1958.
Shukkoor, M.S.A., Bin Baharuldin, M.T.H., Mat Jais, A.M., Moklas M.A.M. \& Fakurazi S., 2016, Antidepressant-Like Effect of Lipid Extract of Channa striatus in Chronic Unpredictable Mild Stress Model of Depression in Rats, EvidenceBased Complementary and Alternative Medicine 2016, 1-17

Slattery, D.A. \& Cryan, J.F., 2012, Using the rat forced swim test to assess antidepressant-like activity in rodents, Nature Protocols 7(6),10091014.

Srivastava, K.K. \& Kumar, R., 2015, Stress, oxidative injury and disease, Indian Journal of Clinical Biochemistry 30(1), 3-10.

Tiller, J.W.G., 2013, Depression and anxiety, Medical Journal of Australia 199 (6 Suppl), S28-S31.

Ulrich-Lai, Y.M., Figueiredo, H.F., Ostrander, M.M., Choi, D.C., Engerland, W.C. \& Herman, J.P., 2006, Chronic stress induces adrenal hyperplasia and hypertrophy in a subregionspecific manner, American Journal of PhysiologyEndocrinology and Metabolism 291(5), 965-73.

Wang, S., Hu, S., Zhang, C., Qiu, J. \& Li, Y., 2014, Antidepressant-like activity of Chaihu-ShuganSan aqueous extract in rats and its possible mechanism, Pharmacognosy Magazine 10(Suppl 1), 50-56.

WHO, 2018, 'Depression', viewed 23 March 2019, from https://www.who.int/news-room/factsheets/detail/depression.

Yau, Y.H.C \& Potenza, M.N, 2014, Stress and Eating Behaviors, Minerva Endocrinology 38(3), 255-267.

Zhang, L., Luo, J., Zhang, M., Yao, W., Ma, X. \& Yu, S.Y., 2014, Effects of curcumin on chronic, unpredictable, mild, stress-induced depressive-like behavior and structural plasticity in the lateral amygdala rats, International Journal of Neuropsychopharmacology 17 (5), 793-806.

Zheng H., Yin, J., Gao, Z., Huang, H., Ji, X. \& Dou, C., 2011, Disruption of Chlorella vulgaris cells for the release of biodiesel-producing lipids: a comparison of grinding, ultrasonication, bead milling, enzymatic lysis, and microwaves, Applied Biochemistry and Biotechnology 164, 121524.

Zoladz, P.R., Fleshner, M. \& Diamond, D.M., 2013, Differential effectiveness of tianeptine, clonidine and amitriptyline in blocking traumatic memory expression, anxiety and hypertension in an animal model of PTSD, Progress in Neuro-Psychopharmacology \& Biological Psychiatry 44, 1-16. 